\title{
CORRIGENDUM \\ Ultrasound-assisted non-viral gene transfer to the salivary glands
}

\author{
MJ Passineau, L Zourelias, L Machen ${ }^{1}$, PC Edwards and RL Benza
}

Gene Therapy (2011) 18, 424; doi:10.1038/gt.2011.30

\author{
Correction to: Gene Therapy (2010) 17, 1318-1324; doi: 10.1038/ \\ gt.2010.86
}

Since the publication of this paper, the authors have noticed that there are several instances in which micrograms were published as milligrams in the Methods section. The correct Methods section is given below.

\section{METHODS}

Microbubble/plasmid solution and viral vectors

Definity microbubbles were purchased as $2 \mathrm{ml}$ injectable suspension and activated with a Vialmix device according to manufacturer's instructions (Lantheus Medical Imaging, North Billerica, MA, USA). Microbubbles were used for experiments within $30 \mathrm{~min}$ of their activation. A total of $50 \mu \mathrm{g}$ of pCMV-GL3 (a GL3 Luc-expressing plasmid constructed by the insertion of a cytomegalovirus (CMV) promoter into the multiple cloning site of pGL3-basic; Promega, Madison, WI, USA) was mixed with $50 \mu \mathrm{g}$ of microbubble solution, either 100 or $15 \%$ Definity solution v/v with phosphate-buffered saline.

For Ad-mediated gene transfer, a non-replicative Ad Serotype 5 vector based on the Stratagene AdEasy system was created by cloning the GL3 sequence (from the Promega pGL3 Basic vector) into the multiple cloning site of the pShuttle-CMV vector (Stratagene, La Jolla, CA, USA). The shuttle vector was recombined into the AdEasy backbone, and the resultant vector, Ad-CMVGL3, was upscaled and purified to a titer of $\sim 3.5 \times 10^{12} \mathrm{vp} \mathrm{ml}^{-1}$. Ad vectors were delivered in $50 \mu \mathrm{g}$ of phosphate-buffered saline.

\section{Animals and salivary gland catheterizations}

Mice of the strain C57BL/6 were obtained from Jackson Labs (Bar Harbor, ME, USA) and maintained in pathogen-free conditions in the Allegheny-Singer Research Institute vivarium, with an access to standard chow and water ad libitum. Animal experiments and protocols were reviewed and approved by the Institutional Animal Care and Use Committee of Allegheny-Singer Research Institute. Gene transfer to the salivary gland is accomplished as previously described by Voutetakis and colleagues. ${ }^{4}$ Briefly, the animal was anesthetized and placed in a stereotactic frame allowing the mouth to be held open. The tongue was retracted and a thin plastic catheter was placed into the opening of the submandibular duct on one side, advanced $\sim 1 \mathrm{~cm}$ and held in place with superglue. A total of $50 \mu \mathrm{g}$ of vehicle solution carrying the gene transfer vector (virus or plasmid/microbubbles) was infused into the duct on one side via the catheter and the plunger was left in place for $10 \mathrm{~min}$ to allow the vector to contact the salivary epithelial cells. The catheter was then removed and the animal was returned to its cage to awaken normally.

For ultrasound gene transfer experiments, the skin immediately overlying the salivary gland was treated with a depilatory agent, a commercial ultrasound gel was applied and an ultrasound emitter (SoniGene, VisualSoincs Inc., Toronto, Ontario, Canada) was placed in direct contact with the skin overlying the gland. After the plasmid microbubble solution was infused, $4 \times 30$-s bursts were applied at the following parameters: $1 \mathrm{MHz}, 50 \%$ duty cycle and $2 \mathrm{~W} \mathrm{~cm}^{-2}$, with $10 \mathrm{~s}$ between pulses. Following the four pulses, the emitter was withdrawn and the animal was allowed to rest for $10 \mathrm{~min}$ before the catheter was removed.

\section{In vivo Luc imaging}

The mice were injected intramuscularly with $1 \mathrm{ml}$ per g of body weight of ketamine $\left(20 \mathrm{~g} \mathrm{ml}^{-1}\right) / x y l a z i n e\left(100 \mathrm{mg} \mathrm{ml}^{-1}\right)$ mixed in the proportion of 3:2. After the mouse was anesthetized, the D-Luciferin substrate (XenoLight D-Luciferin Potassium Salt, Caliper Life Sciences, Hopkinton, MA, USA) was injected intraperitoneally at a dose of $(100 \mu \mathrm{l}$ per $10 \mathrm{~g}$ body weight, stock $15 \mathrm{mg} \mathrm{ml}^{-1}$ ). The mice were then placed in a light-tight chamber, and images were generated over a 1-min exposure using a cryogenically cooled chargecoupling device camera IVIS Lumina II (Caliper Life Sciences) to quantify photons spontaneously emitted by the animal. Images were pseudocolored using the Xenogen (Caliper Life Sciences) software and overlayed on a black-and-white photograph of the animal generated with cabinet lighting. The visual output represents the number of photons emitted per $s$ per $\mathrm{cm}^{2}$ as a pseudocolor image in which the maximum is red and the minimum is purple.

\section{Quantitation of gene transfer and statistical analysis}

Images obtained from in vivo imaging were analyzed using the Living Image software (Caliper Life Sciences). Photons emitted from the salivary gland were quantified by defining an ROI over the anatomical position of the salivary gland. In most cases, this ROI was generated automatically by the software using 'Auto Contour ROI', and total flux (photons per s) in the ROI was measured. In cases where the software was unable to generate an auto ROI, or when no discernable signal was present, a circle with a diameter corresponding to $\sim 0.75 \mathrm{~cm}$ was placed over the anatomical position of the salivary gland, and total flux was measured. We consistently found that $\sim 3 \times 10^{4}$ was the background value in this system. However, values were tabulated as total flux, without a correction for background.

\section{Histology}

To explore any potential differences in sialoadenitis elicited by the two gene delivery methods in the acute setting, we performed gene transfer on a second cohort of animals using methods identical to that described above, with the exception that half of the animals in this cohort were females, with genders being evenly distributed between Ad and UAGT groups. Animals were killed after 7 days and salivary glands were removed, fixed, paraffin embedded, microtome sectioned, and stained with hematoxylin and eosin. A blinded oral pathologist (PCE) examined the slides and graded the degree of sialoadenitis on a 1 (normal) to 4 (severe, with complete destruction of acinar cells) scale on the basis of a modification of Isacsson et al..$^{24}$

To explore the spatial distribution of both plasmid/microbubble vectors and resultant transgene expression, we delivered pCMV-GFP, a plasmid expressing GFP, in the same manner as described above using a $15 \%$ microbubble carrier solution. Immediately before infusion of the plasmid/microbubble mixture and thereafter, ultrasound images of the salivary gland were acquired on a VisualSonics Vevo 770 (VisualSonics Inc., Toronto, Ontario, Canada) with an RMV-704 scanhead running in B-mode at $40 \mathrm{MHz}$. After 7 days, animals were killed and salivary glands were removed, fixed and paraffin embedded. Sections were probed with a fluorescein isothiocyanate-conjugated anti-GFP polyclonal antibody (Abcam, Cambridge, MA, USA).

The authors would like to apologize for this error. 